\title{
Theoretical study of isolated attoseond pulse generation with two methods
}

\author{
Chang-Long Xia *and Xiang-Yang Miao \\ College of Physics and Information Engineering, Shanxi Normal University - Linfen \\ 041004, People's Republic of China
}

Received 10 October 2015; Accepted 13 November 2015

Published Online 1 March 2016

PACS: $32.80 . \mathrm{Rm}, 42.65 . \mathrm{Ky}$

\begin{abstract}
We theoretically investigated high-order harmonic generation and isolated attosecond pulse generation from a model of helium atom by two methods: numerically solve time dependent Schrödinger equation (TDSE) by splitting-operator method and Lewenstein's strong field approximation theory. A left circularly polarized pulse $(800 \mathrm{~nm})$ is combined to a right circular polarized pulse $(1200 \mathrm{~nm})$ with a timedelay of $4 \mathrm{fs}$. A supercontinuum spectrum plateau with a broad bandwidth of $215 \mathrm{eV}$ (from 230 to $445 \mathrm{eV}$ ) is obtained for the case of $I_{0}=7 \times 10^{14} \mathrm{~W} / \mathrm{cm}^{2}$. By superposing a bandwidth of $70 \mathrm{eV}$ in the plateau region, an linear polarized isolated attosecond pulse with the duration of about 56 as can be obtained. Moreover, we illustrate the quantum path control in terms of the time-frequency analysis by Morlet wavelet transform method.
\end{abstract}

Key words: isolated attosecond pulse, high-order harmonic generation, quantum path control

\section{Introduction}

Isolated attosecond pulse (IAP) generation has been a hot topic due to its potential application in ultrafast science [1-3]. Recently, IAPs are mainly generated by superposing high-order harmonic spectra which obtained from intense laser field interacting with atoms or molecules $[4,5]$. The spectrum of high-order harmonic generation (HHG) has a general structure: the intensity decreases rapidly in low order region, and then comes a plateau area, a cutoff with the maximum energy $I_{p}+3.17 U_{p}$ appears at the end, where $I_{p}$ is the ionization potential and $U_{p}=E^{2} / 4 \omega^{2}$ denotes the ponderomotive energy.The feature can't be explained by perturbation theory but can be well explained by the semiclassical three-step model [6]. First, the electron is ionized by tunneling the potential barrier, this is a quantum process. Second, the ionized electron which is regard as free

${ }^{*}$ Corresponding author. Email address: xiach12008@163.com (C.-L. Xia) 
classical electron propagates in the laser field. Finally, as the intense laser field reverse, the electron may go back to the nuclei and emits an energetic photon. In addition, the isolated attosecond pulse would be more useful in pump-probe ultrafast physical process. So control the quantum path that contributing to HHG process is an essential issue.

Different schemes have been proposed to control the quantum path and obtain IAPs, such as few-cycle scheme [4,7], two-color scheme [8-10], three-color scheme, polarization gating (PG) scheme [11] and so on. The PG scheme can be obtained by combing two counterrotating circularly polarized laser pulses with a proper delay, the frequencies of the two pulses are the same. In addition, an IAP with the duration of 67 as was obtained by the double optical gating (DOG) in experiment [12]. The DOG scheme can be obtained by adding another pulse to a PG scheme, this means that the frequency of the two circularly polarized laser pulse is equal . In our previous work [13], we investigated the IAP generation by two circularly polarized pulses with different frequencies, and found that the quantum paths can be controlled. In this paper, we theoretically study the IAP by combining a left circularly polarized pulse with a right circular polarized pulse, and the frequencies are different for the two pulses. To ensure our calculation, we investigate the HHG with two methods: splitting-operator (SO) method [14] and strong field approximation (SFA) model [15].

\section{Theoretical methods}

The laser-matter interaction can be described by Schrödinger equation. Our calculation is based on the single-active electron approximation, and the time dependent Schrödinger equation (TDSE) can be written as (in atomic units)

$$
i \frac{\partial}{\partial t} \Psi(\vec{r}, t)=\left[-\frac{1}{2} \nabla^{2}+U(\vec{r}, t)\right] \Psi(\vec{r}, t),
$$

where, $U(\vec{r}, t)=V(\vec{r})+\vec{r} \cdot \vec{E}(t)$ is the total potential of the Coulomb potential and the lasermatter interaction potential. In this paper, we investigate the HHG by two-color circular polarized laser pulses in two methods.

\section{A. Numerically solve TDSE by SO method}

Because the laser pulse is circular polarized, and it has two dimensions. We numerically solve two-dimensional TDSE:

$$
i \frac{\partial}{\partial t} \Psi(x, y, t)=\left[\frac{p_{x}^{2}+p_{y}^{2}}{2}+U(x, y, t)\right] \Psi(x, y, t),
$$

where, $\left(p_{x}^{2}, p_{y}^{2}\right)$ is the electronic momentum, $U(x, y, t)=V(x, y)+x E_{x}(t)+y E_{y}(t)$ is the total 
potential, and $V(x, y)=-\frac{b}{\sqrt{x^{2}+y^{2}+a}}$ is the "soft-core" Coulomb potential. The TDSE has the formal solution:

$$
\Psi\left(x, y, t_{0}+\Delta t\right)=e^{-i\left(\frac{p_{x}^{2}+p_{y}^{2}}{2}+U\right) \Delta t} \Psi\left(x, y, t_{0}\right),
$$

The part of kinetic energy and the part of potential energy could be splitted, and the solution can be written by second-order splitting-operator fast Fourier transform algorithm,

$$
\Psi\left(x, y, t_{0}+\Delta t\right)=e^{-i\left(\frac{p_{x}^{2}+p_{y}^{2}}{4}\right) \Delta t} e^{-i U \Delta t} e^{-i\left(\frac{p_{x}^{2}+p_{y}^{2}}{4}\right) \Delta t} \Psi\left(x, y, t_{0}\right)+\mathbf{O}(\Delta t)^{3},
$$

In the calculation, from an initial wave function $\Psi\left(x, y, t_{0}\right)$, four-steps are needed to obtain the next moment wave function $\Psi\left(x, y, t_{0}+\Delta t\right)$.First, use the fast Fourier transform (FFT) to change the wave function to momentum space, the part of kinetic energy can be multiplied directly. Second, use the inverse fast Fourier transform (IFFT) to change the wave function back to coordinate space, the part of potential energy can be multiplied directly. The third step is the same as the first step, and the last step is that use IFFT to change the wave function back to coordinate space, and we obtain the wave function $\Psi\left(x, y, t_{0}+\Delta t\right)$. Use the above mentioned four-steps repeatedly until the end of laser pulse, we can get the time-dependent wave function $\Psi(x, y, t)$. The initial wave function is the ground state which is calculated by the imaginary time-propagation method.

We can obtain the time-dependent dipole acceleration by the Ehrenfest theorem as follows,

$$
\begin{aligned}
\vec{a}(t) & =\langle\Psi(x, y, t)|-\vec{\nabla} V(x, y, t)-\vec{E}(t)| \Psi(x, y, t)\rangle \\
& =a_{x}(t) \vec{e}_{x}+a_{y}(t) \vec{e}_{y},
\end{aligned}
$$

The HHG power spectrum is propotional to the modulus squared of the Fourier transformation of $\vec{a}(t)$,

$$
S(\omega) \sim\left|\int \exp (-i \omega t)\left[a_{x}(t) \vec{e}_{x}+a_{y}(t) \vec{e}_{y}\right] d t\right|^{2},
$$

The temporal profile of an IAP can be obtained by superposing several harmonics, in the $\mathrm{x}$ and $\mathrm{y}$ compones:

$$
I_{x, y}(t)=\left|\sum a_{x, y} e^{i q \omega t}\right|^{2}
$$




\section{B. The Lewenstein's SFA model}

To calculate the HHG spectrum, Lewenstein proposed SFA model: i) the contribution to the HHG mainly come from the ground state, ii) the depletion of the ground state can be neglected, iii) in the continuum, the electron can be treated as a free particle. Base on the above approximation, the instantaneous dipole moment of an atom can be described as,

$$
\begin{aligned}
\vec{r}= & i \int_{0}^{\infty} d \tau\left(\frac{\pi}{\varepsilon+i \pi / 2}\right)^{\frac{3}{2}} d^{*}\left[\vec{p}_{s}(t, \tau)-\vec{A}(t)\right] e^{-i S(\vec{p}, t, \tau)} \\
& \times \vec{E}(t-\tau) \cdot \vec{d}\left[\vec{p}_{s}(t, \tau)-\vec{A}(t-\tau)\right]+\text { c.c. }
\end{aligned}
$$

where $\vec{A}(t)$ is the vector potential, $\varepsilon$ is a small number, $\vec{p}_{s}(t, \tau)=\int_{t-\tau}^{t} d t^{\prime \prime} \vec{A}\left(t^{\prime \prime}\right) / \tau$ is the canonical momentum of the electron corresponding to a stationary phase. $S\left(\vec{p}, t, t^{\prime}\right)$ is the quasiclassiical action of the electron. $\vec{d}\left[\vec{p}_{s}-\vec{A}(t)\right]$ is the field-free dipole transition matrix element between the ground state and the continuum state.

In our calculation, the evolution of the laser pulse is in xy plane, the dipole moment is calculated y the integral as follows,

$$
\begin{aligned}
x(t)= & i \int_{0}^{\infty} d \tau\left(\frac{\pi}{\varepsilon+i \pi / 2}\right)^{\frac{3}{2}} d_{x}^{*}\left[\vec{p}_{s}(t, \tau)-\vec{A}(t)\right] e^{-i S(\vec{p}, t, \tau)} \\
& \left\{E_{x}(t-\tau) \cdot d_{x}\left[\vec{p}_{s}(t, \tau)-\vec{A}(t-\tau)\right]+E_{y}(t-\tau) \cdot d_{y}\left[\vec{p}_{s}(t, \tau)-\vec{A}(t-\tau)\right]\right\} \\
y(t)=i \int_{0}^{\infty} d \tau\left(\frac{\pi}{\varepsilon+i \pi / 2}\right)^{\frac{3}{2}} d_{y}^{*}\left[\vec{p}_{s}(t, \tau)-\vec{A}(t)\right] e^{-i S(\vec{p}, t, \tau)} & \\
& \left\{E_{x}(t-\tau) \cdot d_{x}\left[\vec{p}_{s}(t, \tau)-\vec{A}(t-\tau)\right]+E_{y}(t-\tau) \cdot d_{y}\left[\vec{p}_{s}(t, \tau)-\vec{A}(t-\tau)\right]\right\}
\end{aligned}
$$

where,

$$
\begin{aligned}
& d_{x}^{*}\left[\vec{p}_{s}(t, \tau)-\vec{A}(t)\right]=-i \frac{2^{7 / 2}}{\pi} \alpha^{5 / 4} \times \frac{p_{s, x}(t, \tau)-A_{x}(t)}{\left\{\left[p_{s, x}(t, \tau)-A_{x}(t)\right]^{2}+\left[p_{s, y}(t, \tau)-A_{y}(t)\right]^{2}+\alpha\right\}^{3}} \\
& d_{y}^{*}\left[\vec{p}_{s}(t, \tau)-\vec{A}(t)\right]=-i \frac{2^{7 / 2}}{\pi} \alpha^{5 / 4} \times \frac{p_{s, y}(t, \tau)-A_{y}(t)}{\left\{\left[p_{s, x}(t, \tau)-A_{x}(t)\right]^{2}+\left[p_{s, y}(t, \tau)-A_{y}(t)\right]^{2}+\alpha\right\}^{3}} \\
& S\left(\vec{p}_{s, t}, \tau\right)=I_{p}-\frac{1}{2}\left[p_{s, x}^{2}(t, \tau)+p_{s, y}^{2}(t, \tau)\right]+\frac{1}{2} \int_{t-\tau}^{t} d t^{\prime \prime}\left[A_{x}^{2}\left(t^{\prime \prime}\right)+A_{y}^{2}\left(t^{\prime \prime}\right)\right]
\end{aligned}
$$


and $\alpha=2 I_{p}, p_{s, x(y)}(t, \tau)=\int_{t-\tau}^{t} d t^{\prime \prime} A_{x(y)}\left(t^{\prime \prime}\right) / \tau$. The time-dependent dipole acceleration $\vec{a}(t)=$ $\ddot{x}(t) \vec{e}_{x}+\ddot{y}(t) \vec{e}_{y}$, the HHG spectrum and the temporal profile of attosecond pulse can be obtained by Eq. (6) and Eq.(7).

In this paper, we theoretically investigate the high-order harmonic generation (HHG) in a two-color circular polarized laser pulses. The combination of the pulse with a timedependent ellipticity is generated by the superposition of a left and a right circular polarized Gaussian pulse. The electric fields propagating in the $z$ direction are

$$
\begin{aligned}
& \vec{E}_{l}(t)=E_{0} f\left(t-T_{d} / 2\right)\left[\cos \left(\omega_{1} t\right) \hat{x}+\sin \left(\omega_{1} t\right) \hat{y}\right] \\
& \vec{E}_{r}(t)=E_{0} f\left(t+T_{d} / 2\right)\left[\cos \left(\omega_{2} t+\varphi\right) \hat{x}+\sin \left(\omega_{2} t+\varphi\right) \hat{y}\right]
\end{aligned}
$$

where $E_{0}$ is the peak field amplitude, $f(t)=e^{-2 \ln 2 t^{2} / \tau^{2}}$ is the envelope of the laser field, $\tau=$ $5 f s$ is the pulse duration and $T_{d}=4 f s$ is the time delay between two pulses. $\omega_{1}=0.057$, $\omega_{2}=0.038$ are the frequencies of the two pluses that corresponding to the wavelength of $800 \mathrm{~nm}$ and $1200 \mathrm{~nm}$, the $\hat{x}$ and $\hat{y}$ are unit vectors in the $x$ and $y$ directions, respectively. The electric field of the combined pulse is $\vec{E}(t)=\vec{E}_{l}(t)+\vec{E}_{r}(t)$.

\section{Results and discussion}

First, we set the intensity $I_{0}=3 \times 10^{14} \mathrm{~W} / \mathrm{cm}^{2}$ and change the carrier envelope phase to investigate the HHG. The HHG spectra are show in Fig. 1 for the case of $\varphi=0$ and $\varphi=0.5 \pi$, Fig. 1(a) and Fig. 1(b) are obtained with SFA model and SO method respectively. The structures of the HHG are similar from the two methods. The intensity of the HHG spectrum decreases quickly at the low energy area, and then comes a plateau structure for the two cases. Modulations are all around in plateau area for the case of $\varphi=0$, and the cut of is about $195 \mathrm{eV}$. For the case of $\varphi=0.5 \pi$, a smooth structure is obtained near the cut off area, and the cut off of the HHG extend to $205 \mathrm{eV}$, but the intensity is lower than the case of $\varphi=0$. One of the difference with our previous work [13] is that the cut off extend for the case of $\varphi=0.5 \pi$, but the result shown in Ref. [13] (Fig. 2) is for the case of the wavelength equal $800 \mathrm{~nm}$ for the two circular pulses. The modulations may come from the quantum interference and this is disadvantageous to generation IAP.

We calculate the time-frequency analysis by Morlet wavelet transform method to further investigate the emission time of the HHG. For the case of $\varphi=0$, Fig. 2(a) shows three peaks contributing to the $\mathrm{HHG}$, and the peak $B_{1}$ contains both long and short quantum path, the intensity of long quantum is weaker than the short one. The energy of peak $A_{1}$ and peak $C_{1}$ is low, which is contributing to the low order of HHG. Fig. 2(b) shows for the case of $\varphi=0.5 \pi$, the peak $C_{2}$ is too weak to be ignored, the energy of peak $A_{2}$ is increase but the intensity is also weaker than the peak $B_{2}$, the long quantum path is suppressed. The contribution for the plateau area of HHG is mainly come from the short quantum path. This can explain the modulations is less in Fig. 1 for the case of $\varphi=0.5 \pi$. 

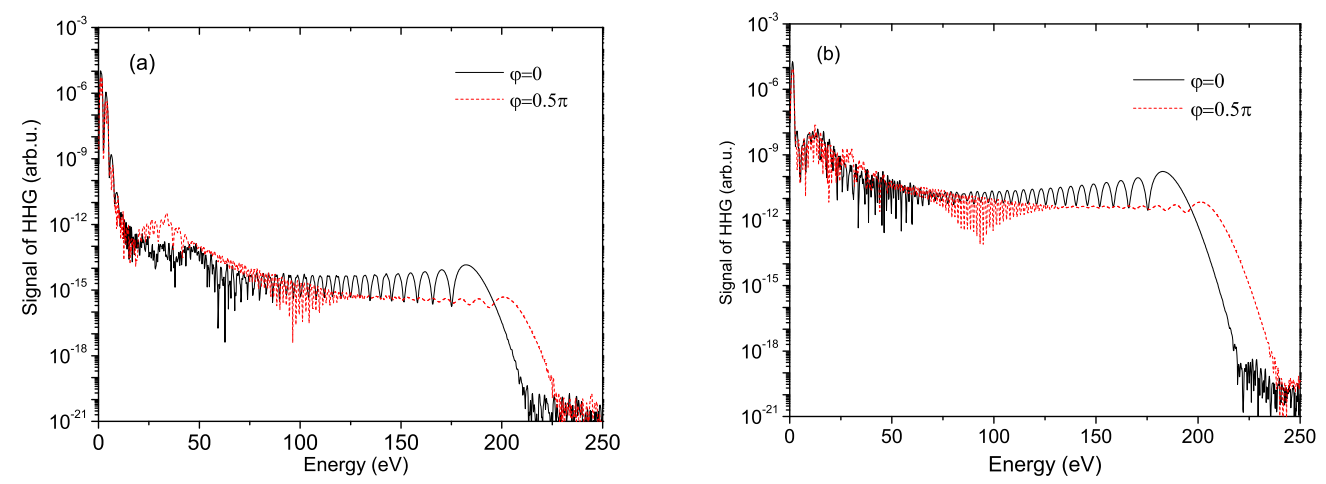

Figure 1: (Color online)The HHG spectra for the case of $\varphi=0$ and $\varphi=0.5 \pi$. (a) by the SFA model, (b) by SO method.
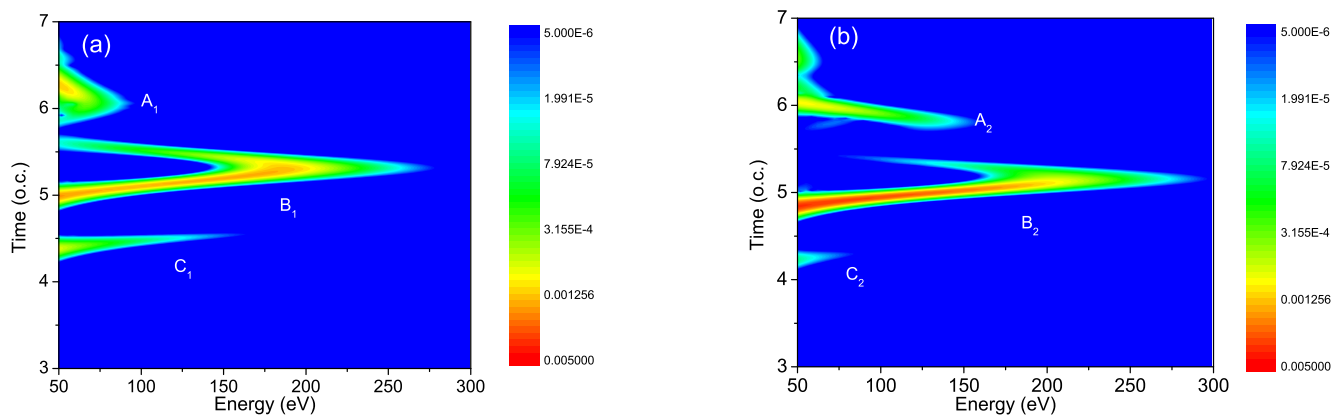

Figure 2: (Color online) The time-frequency analysis for the cases: (a) $\varphi=0$, (b) $\varphi=0.5 \pi$. The parameters are the same as shown in Fig. 1.

To better control the quantum path and eliminate the interference from the quantum paths, we increase the intensity of the laser pulses, the results form SFA model is shown in Fig. 3. From the figure, the cut off are $325 \mathrm{eV}, 445 \mathrm{eV}$ and $615 \mathrm{eV}$ for the cases of $I_{0}=$ $5 \times 10^{14} \mathrm{~W} / \mathrm{cm}^{2}, I_{0}=7 \times 10^{14} \mathrm{~W} / \mathrm{cm}^{2}$ and $I_{0}=1 \times 10^{15} \mathrm{~W} / \mathrm{cm}^{2}$, respectively. The stronger laser pulse may give greater ponderomotive energy, so the cut off increases with increasing the intensity of the laser pulse. The plateaus of the HHG with broad bandwidth of about 135 $\mathrm{eV}$ (from 190 to $325 \mathrm{eV}$ ) and $215 \mathrm{eV}$ (from 230 to $445 \mathrm{eV}$ ) are smooth near the cut off area for the case of $I_{0}=5 \times 10^{14} \mathrm{~W} / \mathrm{cm}^{2}$ and $I_{0}=7 \times 10^{14} \mathrm{~W} / \mathrm{cm}^{2}$, which indicates the interference from the quantum path is weak and is good for IAP generation. Although the plateau is broadest for the case of $I_{0}=1 \times 10^{15} \mathrm{~W} / \mathrm{cm}^{2}$, the modulations are all around the plateau area. The HHG spectra obtaining from SO method are similar but not show here for short. We calculate the time frequency analysis to reveal the quantum paths, Fig. 3 (b) shows the case of $I_{0}=7 \times 10^{14} \mathrm{~W} / \mathrm{cm}^{2}$. There are two peaks marked $A_{3}$ and $B_{3}$ contribute to the HHG, and both the peaks have only short quantum path, the long quantum path 

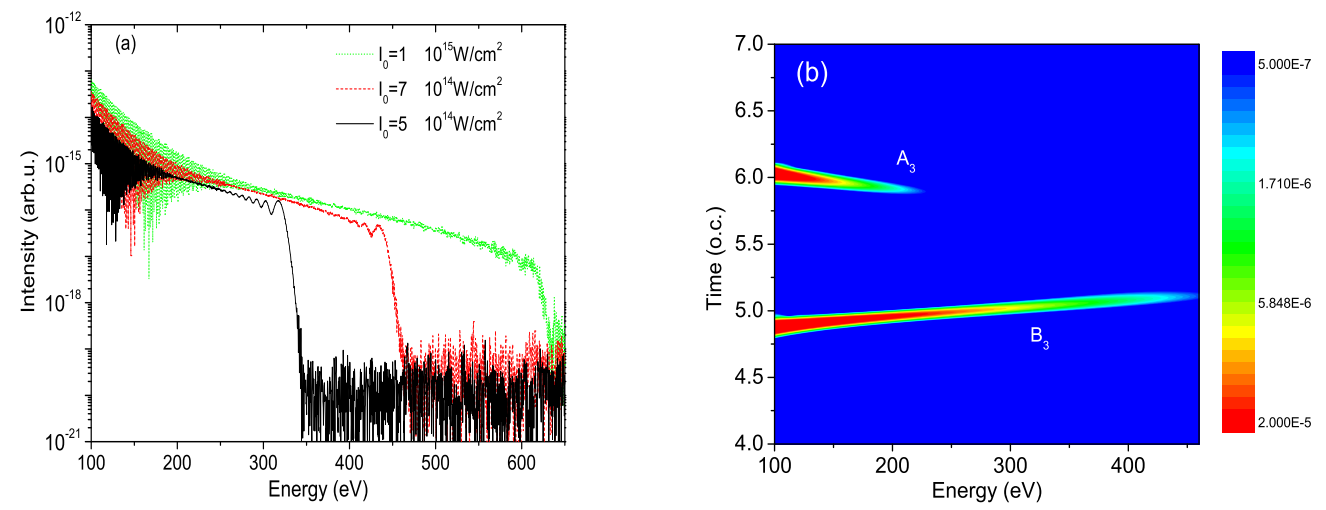

Figure 3: (Color online)(a) The HHG spectra for the cases of $I_{0}=5 \times 10^{14} \mathrm{~W} / \mathrm{cm}^{2}, I_{0}=7 \times 10^{14} \mathrm{~W} / \mathrm{cm}^{2}$ and $I_{0}=1 \times 10^{15} \mathrm{~W} / \mathrm{cm}^{2}$. (b) The time frequency for the case of $I_{0}=7 \times 10^{14} \mathrm{~W} / \mathrm{cm}^{2}$, The phase is $\varphi=0.5 \pi$ and other parameters are the same as shown in Fig. 1.
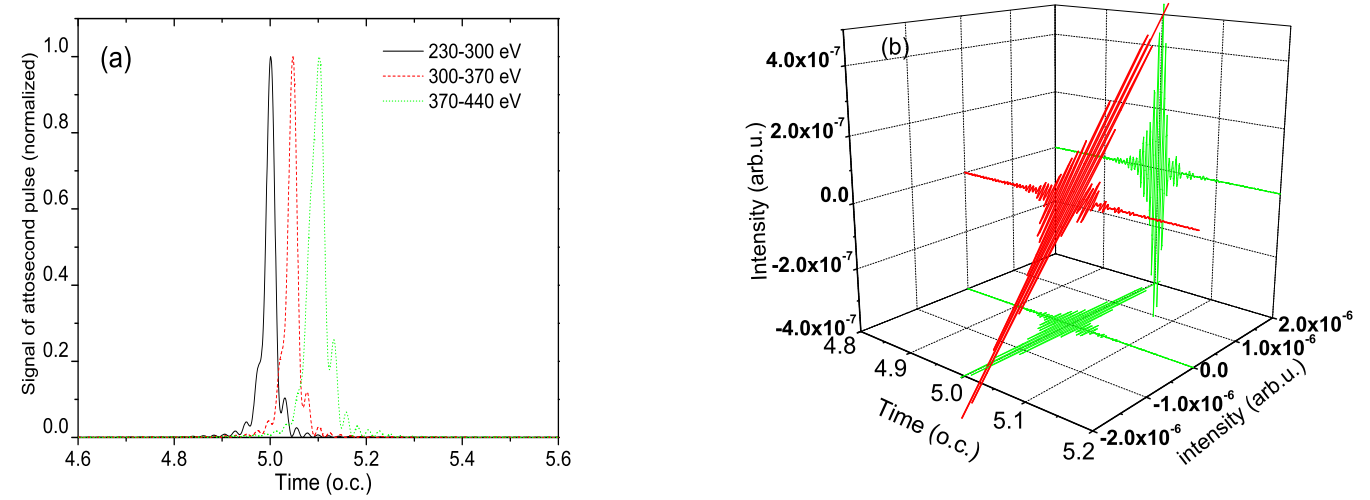

Figure 4: (Color online)(a) IAP obtained by superposing a band width of $70 \mathrm{eV}$ for the case of $I_{0}=7 \times$ $10^{14} \mathrm{~W} / \mathrm{cm}^{2}$, the region of $230-300 \mathrm{eV}, 300-370 \mathrm{eV}$ and $370-440 \mathrm{eV}$ are shown in black solid line, red dashed line and green dotted line respectively. (b) Electric field of IAP in the 3D frame, the parameters are the same as red dashed line shown in Fig. 4(a).

are eliminated. This illustrates that the plateau of HHG spectrum near the cut off area only has one short quantum path. We may use this region to obtain IAP pulse.

At last, we investigate the IAP generation by superposing several orders of the harmonics. The temporal envelopes are obtained by summing up the intensities of the $x$ and the y components and normalized. For the case of $I_{0}=7 \times 10^{14} \mathrm{~W} / \mathrm{cm}^{2}$, we calculate the IAP generation by superposing a width of $70 \mathrm{eV}$ in plateau region near the cut off. As shown in Fig. 4 (a), isolated attosecond pulses with the duration of 56 as, 58 as and 91 as by superposing the harmonics in the range of $230-300 \mathrm{eV}, 300-370 \mathrm{eV}$ and $370-440 \mathrm{eV}$, respectively. The emission time is about 4.9-5.2 o.c., which is corresponding to the emission 
time of quantum path $B_{3}$. For the case of $I_{0}=5 \times 10^{14} \mathrm{~W} / \mathrm{cm}^{2}$, IAP with duration of about 60 as can also be obtained in plateau region, the results are not show here for short. In addition, we calculated the electric fields of the IAP pulse in the three-dimensional (3D) frame axes to reveal the feature of the polarization. Take the case of dashed line in Fig. 4 (a) as an example, Fig. 4 (b) shows the 3D attosecond pulse. A linear polarization IAP pulse is obtained, which implies the phases of attosecond pulse in $x$ direction and in the $y$ direction are synchronous. Linear attosecond is more useful in experiment than the nonpolarized pulse. In a word, we obtain linear polarization IAPs directly by superposing a band width of $70 \mathrm{eV}$ in the plateau region.

\section{Conclusions}

In summary, we theoretically investigated HHG from helium atom by two methods. The scheme is used by two-color circular polarized pulses, which is combined a left circularly polarized pulse with wavelength of $800 \mathrm{~nm}$ to a right circular polarized pulse with wavelength of $1200 \mathrm{~nm}$. A supercontinuum spectrum plateau from 230 to $445 \mathrm{eV}$ is obtained for the case of $I_{0}=7 \times 10^{14} \mathrm{~W} / \mathrm{cm}^{2}$. An linear polarized IAP with duration of about 56 as can be generated by superposing a bandwidth of $70 \mathrm{eV}$ in the plateau region. Moreover, we illustrate the quantum path control in terms of the time-frequency analysis of high-order harmonic generation.

Acknowledgments. This work is supported by National Natural Science Foundation of China (Grant Nos. 11404204, 11447208, 11504221), Natural Science Foundation for Young Scientists of Shanxi Province, China (Grant No. 2015021023), and Program for the Top Young Academic Leaders of Higher learning Institutions of Shanxi Province, China.

\section{References}

[1] Y. Wu and X. X. Yang, Phys. Rev. Lett. 98 (2007) 013601.

[2] S. B. Schoun, R. Chirla, J. Wheeler, et al. Phys. Rev. Lett. 112 (2014) 153001.

[3] K. Krausz and M. Ivanov, Rev. Mod. Phys. 81 (2009) 163.

[4] E. Guolielmakis, M. Schultze, M. Hofsteter et al. Science 320 (2008) 1614.

[5] K. J. Yuan, A. D. Bandrauk, Phys. Rev. Lett. 110 (2013) 023003.

[6] P. B. Corkum, Phys. Rev. Lett. 71 (1993) 1994.

[7] C. Altucci, V. Tosa, and R. Velotta, Phys. Rev. A 75 (2007) 061401(R).

[8] H. Du, L. Luo, X. Wang, and B. Hu, Opt. Express 20 (2012) 9713.

[9] E. J. Takahashi, P. Lan, O. D. Mucke, et al. Phys. Rev. Lett. 104 (2010) 233901.

[10] S. F. Zhao, X. X. Zhou, P. C. Li, et al. Phys. Rev. A 78 (2008) 063404.

[11] Z. Chang, Phys. Rev. A 71 (2015) 023813.

[12] K. Zhao, Q. Zhang, M. Chini et al. Opt. Lett. 37 (2012) 3891.

[13] C. L. Xia, and X. S. Liu, Phys. Rev. A 87 (2013) 043406.

[14] M. D. Feit, Jr. J. A. Fleck, and A. Steiger, J. Comput Phys. 47 (1982) 412.

[15] M. Lewenstein, P. Balcou, Y. Ivanov, et al. Phys. Rev. A 49 (1994) 2117. 